\title{
Metabolomics of the Bio-Degradation Process of Aflatoxin B1 by Actinomycetes at an Initial pH of 6.0
}

\author{
Manal Eshelli ${ }^{1, *}$, Linda Harvey ${ }^{2}$, RuAngelie Edrada-Ebel ${ }^{2}$ and Brian McNeil ${ }^{2, *}$ \\ 1 Food Science and Technology Department, Faculty of Agriculture, University of Tripoli, \\ Tripoli, Libya \\ 2 Fermentation Laboratory, Strathclyde Institute of Pharmacy and Biomedical Sciences, \\ University of Strathclyde, 161 Cathedral Street, Glasgow G4 0RE, UK; \\ E-Mails: L.M.Harvey@ strath.ac.uk (L.H.); ruangelie.edrada-ebel@ strath.ac.uk (R.E.-E.) \\ * Authors to whom correspondence should be addressed; \\ E-Mails: m.eshelli@ @otmail.com (M.E.); b.mcneil@strath.ac.uk (B.M.); \\ Tel.: +44-1412377203 (M.E.); +44-1415484379 (B.M.).
}

Academic Editor: Annie Pfohl-Leszkowicz

Received: 17 November 2014 / Accepted: 23 January 2015 / Published: 4 February 2015

\begin{abstract}
Contamination of food and feed by Aflatoxin B1 (AFB1) is a cause of serious economic and health problems. Different processes have been used to degrade AFB1. In this study, biological degradation of AFB1 was carried out using three Actinomycete species, Rhodococcus erythropolis ATCC 4277, Streptomyces lividans TK 24, and S. aureofaciens ATCC 10762, in liquid cultures. Biodegradation of AFB1 was optimised under a range of temperatures from 25 to $40{ }^{\circ} \mathrm{C}$ and $\mathrm{pH}$ values of 4.0 to 8.0. An initial concentration of $20 \mu \mathrm{g} / \mathrm{mL}$ of AFB1 was used in this study. The amount of AFB1 remaining was measured against time by thin layer chromatography (TLC) and high-performance liquid chromatography (HPLC), coupled with UV and mass spectrometry (LC-MS). All species were able to degrade the AFB1, and no significant difference was found between them. AFB1 remained in the liquid culture for $R$. erythropolis, S. lividans and $S$. aureofaciens were $0.81 \mu \mathrm{g} / \mathrm{mL}$, $2.41 \mu \mathrm{g} / \mathrm{mL}$ and $2.78 \mu \mathrm{g} / \mathrm{mL}$ respectively, at the end of the first $24 \mathrm{~h}$. Degradation occurred at all incubation temperatures and the $\mathrm{pH}$ with the optimal conditions for $R$. erythropolis was achieved at $30{ }^{\circ} \mathrm{C}$ and $\mathrm{pH}$ 6, whereas for S. lividans and $S$. aureofaciens the optimum conditions for degradation were $30{ }^{\circ} \mathrm{C}$ and $\mathrm{pH} 5$. Analysis of the degradative route indicated that each microorganism has a different way of degrading AFB1. The metabolites produced by $R$. erythropolis were significantly different from the other two microorganisms. Products
\end{abstract}


of degradation were identified through metabolomic studies by utilizing high-resolution mass spectral data. Mass spectrometric analysis indicated that the degradation of AFB1 was associated with the appearance of a range of lower molecular weight compounds. The pathway of degradation or chemical alteration of AFB1 was followed by means of high resolution Fourier transform mass spectrometry (HR-FTMS) analysis as well as through the $\mathrm{MS}^{2}$ fragmentation to unravel the degradative pathway for AFB1. AFB1 bio-degradation was coupled with the accumulation of intermediates of fatty acid metabolism and glycolysis. A plausible mechanism of degradation of AFB1 by Rhodococcus was hypothesized.

Keywords: aflatoxin $\mathrm{B}_{1}$; biodegradation; microorganisms; metabolites; high-pressure liquid chromatography tandem mass spectrometry (LC-MS/MS)

\section{Introduction}

Mycotoxin can contaminate food and feed as a result of the growth of fungi before harvesting or because of improper storage [1]. It can be found in almost $25 \%$ of the world's agricultural commodities [2]. Aflatoxins are mycotoxin secondary metabolites and are the most hazardous mycotoxins. Aflatoxins were first isolated and characterized following the death of more than 100,000 turkeys from an unidentified disease. The toxin was traced to consumption of a mould-contaminated peanut meal [1]. Among eighteen different types of aflatoxins identified, the major members are aflatoxin $B_{1}, B_{2}, G_{1}$ and $G_{2}$. AFB1 is the most toxic and usually prevalent in cultures as well as in food products [3]. AFB1 is a difuranocoumarin derivative (Figure 1) and is considered to be heat stable when molecules are within the range of conventional food processing temperatures $\left(80-121^{\circ} \mathrm{C}\right)[4]$. Once the food is contaminated with aflatoxins, there are only two options: either the toxin is removed, or the toxin is degraded into less toxic or non-toxic compounds [5].

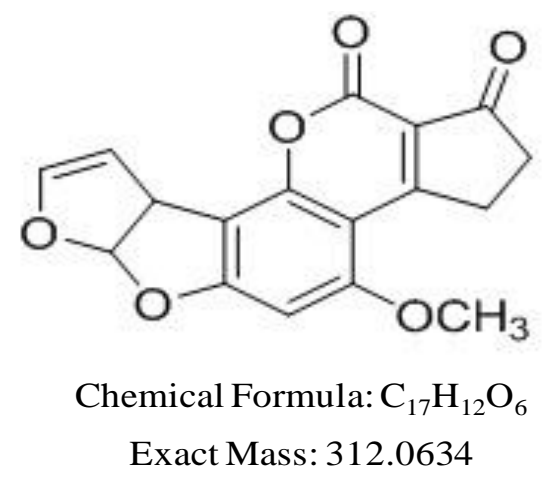

Figure 1. Chemical structure for AFB1.

Different physical and chemical methods have been developed and tested to control AFB1. Disadvantages of these methods are limited in their practical applications due to nutritional losses, sensory quality reduction, and high cost. Ideally, any detoxification procedure should consider reducing the concentration of toxins to safe levels, preventing production of toxic degradation products and avoiding the loss of nutritional value of treated products [6-9]. The application of enzymes 
or microorganisms is an alternative method to detoxify AFB1 in contaminated food and feed [8,10]. A number of fungal species such as Pleurotus ostreatus [11], Rhizopus sp. [6,12], Armillariella tabescens [6], and Trichoderma strains, [13] as well as the yeast Saccharomyces cerevisiae [14] have been found to be able to transform AFB1 into less toxic metabolites. However, the use of fungi and yeasts is not economical because of the extraction process and lengthy incubation time. Reports recorded the reduction of AFB1 by lactic acid bacteria such as Lactobacillus sp. [15], Lactococcus sp. [5,16], and Bifidobacterium sp. [17]. The reduction of AFB1 by lactic acid bacteria was due to AFB1 binding to bacterial cell walls [18]. Therefore, complete toxin removal from contaminated food or feed is difficult or impossible [5,18].

Soil microorganisms were also examined for their ability to degrade AFB1 [15]. The effective bacteria were limited to Actinomycetes bacteria, including R. erythropolis, Mycobacterium fluoranthenivorans, Flavobacterium aurantiacum and Stenotrophomonas maltophilia [19-21]. The efficiency of R. erythropolis culture on AFB1 degradation was first reported in 2006. Rhodococcus are anaerobic, Gram-positive bacterium capable of transforming a wide range of xenobiotic compounds [22]. Rhodococcus has a large genome, and mega-plasmids make them suitable industrially for biotransformation and biodegradation [10]. In addition, Streptomyces also has a long history in the industry of antibiotic production and can metabolize aromatic compounds by producing extracellular enzymes [23,24]. This seems to be a potentially promising prospect for the biodegradation of AFB1 [25,26]. To establish bio-decontamination methods, greater understanding of AFB1 metabolism by these microorganisms is required, particularly in terms of optimizing the biodegradation conditions, pathways, and the function of the respective enzymes involved in the degradation. Therefore, the focus in this study was to investigate the degradation of AFB1 by Actinomycetes liquid cultures and determine the optimum conditions for AFB1 degradation that involves the variable factors: $\mathrm{pH}$, temperature, and incubation time. Additionally, the aim was to identify the degradation products by using the tools of metabolomics, which involved high resolution FTMS (Fourier Transform Mass Spectrometry) and an automated label-free differential expression analysis software (SIEVE).

\section{Results and Discussion}

\subsection{AFB1 Degradation by Actinomyces in Liquid Culture}

Liquid cultures of $R$. erythropolis ATTC 4277, S. lividans TK 24 and S. aureofaciens ATCC10762 were exposed to $20 \mu \mathrm{g} / \mathrm{mL}$ of AFB1 for 24,48 , and $72 \mathrm{~h}$. All strains were able to degrade AFB1 in ISP medium No. 1. As illustrated in Figure 2, a significant reduction of AFB1 to $0.81 \mu \mathrm{g} / \mathrm{mL}$ was observed after $24 \mathrm{~h}$ incubation. This meant that only 4\% of AFB1 remained in the culture with $R$. erythropolis ATCC 4277 at $30{ }^{\circ} \mathrm{C}$ and pH 6.0. Meanwhile, the remaining amount of AFB1 was slightly higher when Streptomyces strains were used under the same conditions: 12\% (S. lividans) and 14\% (S. aureofaciens) of the initial concentration after $24 \mathrm{~h}$ incubation. The control sample (AFB1 + media) was stable over the period degradation in ISP No. 1 media. This study complements the range of soil bacteria studied which has the ability to degrade AFB1 in vitro during incubation. Evidence was revealed in this research for AFB1 degradation by these microorganisms. This was in agreement with Ciegler et al., (1966) [25] who reported that Flavobacterium aurantiacum NRRL B-184, showed a high capacity for degrading of 
AFB1. It was considered to be the only bacteria able to degrade AFB1 [25]. However, recently, other soil microbes have also been tested for degrading AFB1 $[19,26]$. The liquid cultures and cell-free extract of $R$. erythropolis DSM 14303, Nocardia corynebacterioides DSM 12676, N. corynebacterioides DSM 20151, and Mycobacterium fluoranthenivorans sp. nov. DSM 44556T degraded AFB1 effectively [19].

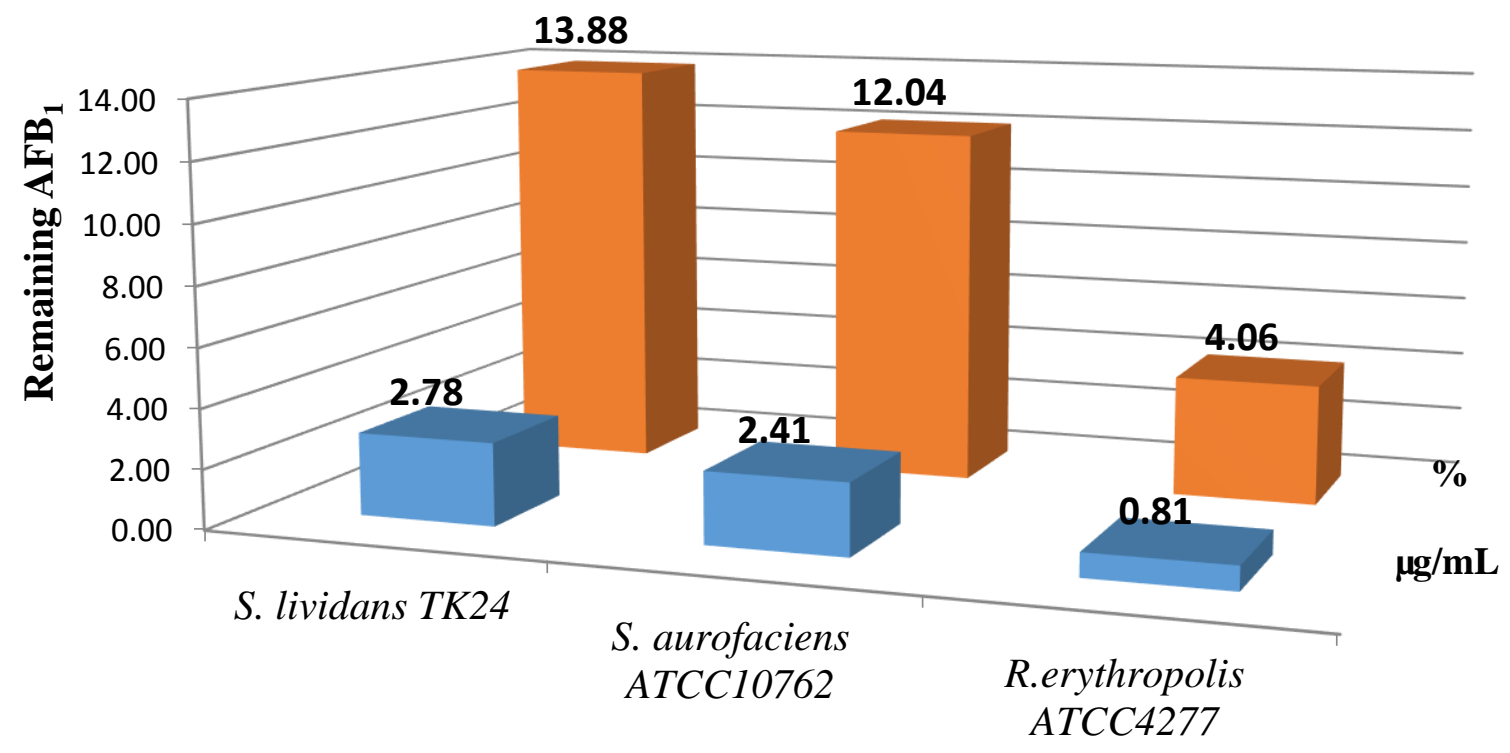

\section{Bacterial Strains}

Figure 2. AFB1 degradation by cultures of R. erythropolis ATCC 4277, S. lividans TK 24 and $S$. aureofaciens ATCC10762 after $24 \mathrm{~h}$ incubation at $30^{\circ} \mathrm{C}$ and $\mathrm{pH} 6.0$.

\subsection{Optimising AFB1 Degradation Conditions}

The effect of different temperatures on AFB1 degradation by Actinomycetes was also studied. A general degradation of AFB1 was observed for all the strains at different temperatures. The optimal degradation temperature was $30{ }^{\circ} \mathrm{C}$ at $\mathrm{pH} 6.0$ for all the strains. In addition, Streptomyces strains had no significant difference in AFB1 degradation between 25 and $30{ }^{\circ} \mathrm{C}$ (Figure 3). This may be due to the range of enzymes produced from these microorganisms or the compatibility of their enzymes to work at a wide range of temperatures. This result agrees with Guan et al., (2008) [22] as they recorded that no significant difference was observed between 20 and $30{ }^{\circ} \mathrm{C}$ when S. maltophilia 35-3 was used to degrade AFB1. The results in this study showed that the optimal degradation temperature was at $30{ }^{\circ} \mathrm{C}$ and $\mathrm{pH} 6.0$ for $R$. erythropolis ATCC 4277, while the optimal degradation temperature for Streptomyces strains was $30{ }^{\circ} \mathrm{C}$ and $\mathrm{pH} 5.0(p<0.05)$. In another work, the optimum temperature for Flavobacterium aurantiacum to break down AFB1 was $25^{\circ} \mathrm{C}[19,25]$ whereas no significant differences were found between temperature 10 and $40{ }^{\circ} \mathrm{C}$ in terms of AFB1 degradation by using the cell-free extracts of $R$. erythropolis and M. fluoranthenivorans [19]. 


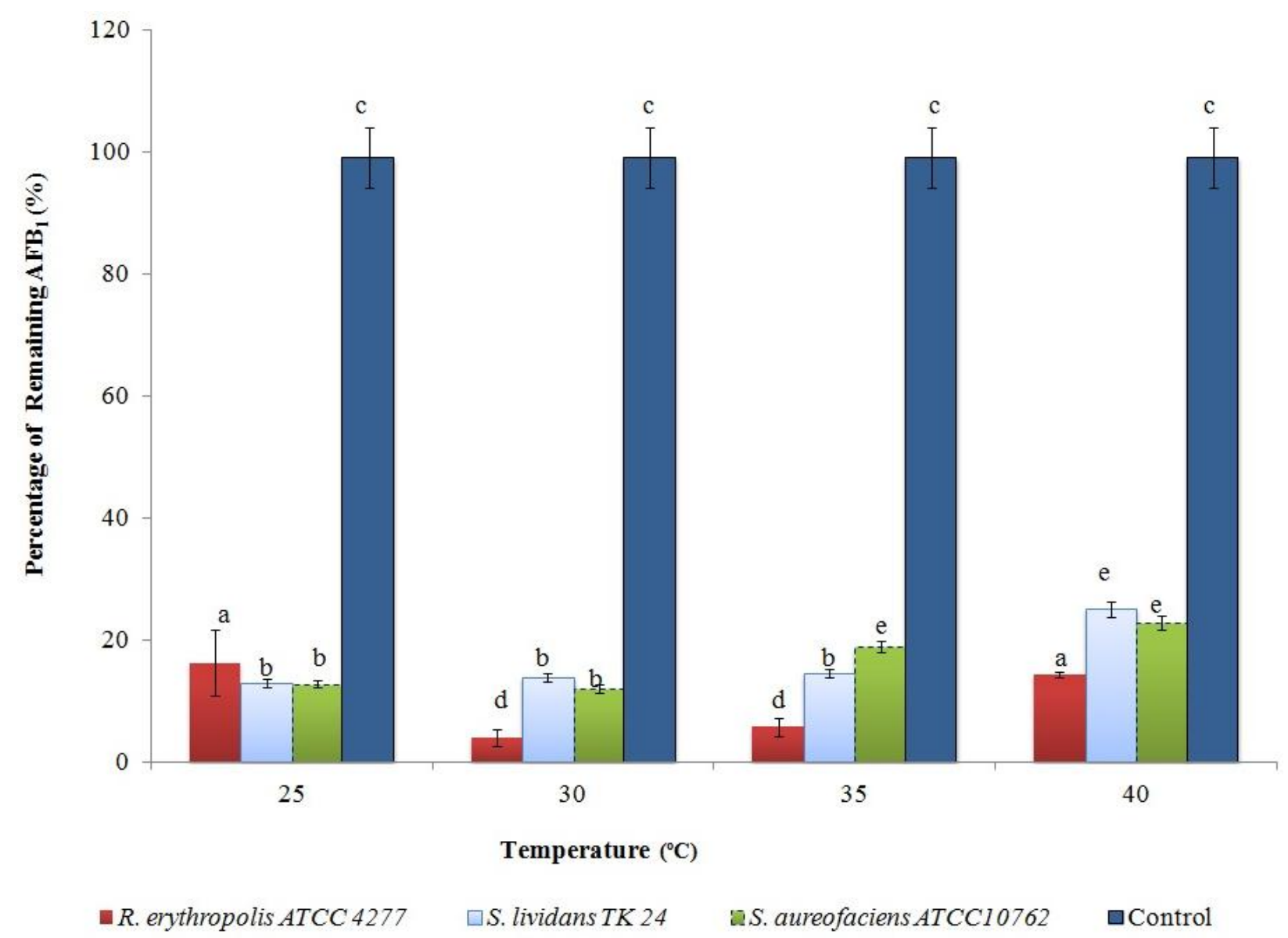

Figure 3. The effect of incubation temperature on AFB1 degradation over the first $24 \mathrm{~h}$ of culture at $\mathrm{pH}$ 6.0. The values are the mean of three replicates and their standard errors. Means with different letters are significantly different according to the Bonferroni Multiple Comparisons Test $(p<0.05)$.

The effect of initial culture $\mathrm{pH}$ on AFB1 degradation over the first $24 \mathrm{~h}$ incubation at $30{ }^{\circ} \mathrm{C}$ was examined (Figure 4). The results indicated that AFB1 was not stable in all the pHs used in this study. The most efficient degradation was around a natural $\mathrm{pH}$ with an optimum of degradation as no significant differences were found between $\mathrm{pH} 6.0$ and $\mathrm{pH} 7.0$ for $R$. erythropolis. In contrast, the optimum degradation for Streptomyces was achieved at $\mathrm{pH}$. The initial $\mathrm{pH}$ did not have any significant effect on the amount of AFB1 in the control over a period of $24 \mathrm{~h}$. This was in agreement with Ciegler et al., (1966) [25] whose result showed the optimum $\mathrm{pH}$ for AFB1 degradation by Flavobacterium aurantiacum was at $\mathrm{pH} 6.75$ and the rate of AFB1 degradation was decreased at $\mathrm{pH} 5$ and 8, respectively. In another study, the optimum degradation of AFB1 by Flavobacterium auranticum was observed at $\mathrm{pH} 7$, with some AFB1 degradation occurring at $\mathrm{pH}$ levels as low as 5 and as high as 8 [27]. However, the optimum degradation of extracellular enzymes of Pleurotus ostreatus was achieved at $\mathrm{pH} 4$ and $\mathrm{pH} 5$, and at $25^{\circ} \mathrm{C}$ activities were found at $\mathrm{pH} 4$ and $\mathrm{pH} 5$ [11]. Meanwhile, Guan et al. (2008) [21] reported that the maximum AFB1 degradation by $S$. maltophilia 35-3 was at pH 8. However, identifying the optimum conditions and understanding the underlying key factors involved in the biodegradation process may assist in developing a model for use on an industrial Fermentation scale. 


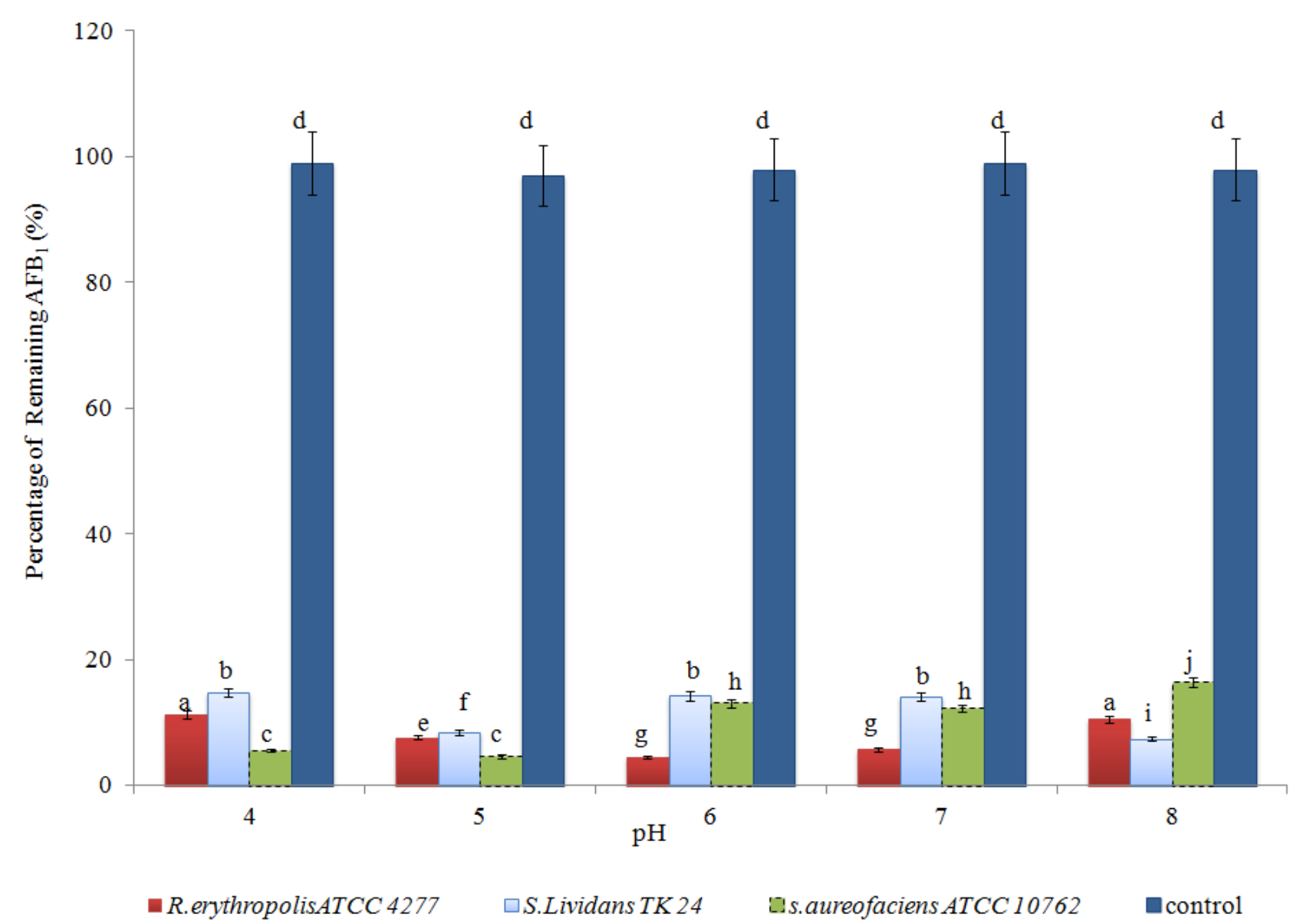

Figure 4. The effect of culture $\mathrm{pH}$ on AFB1 degradation at $30{ }^{\circ} \mathrm{C}$ over the first $24 \mathrm{~h}$ of incubation. Means with different letters are significantly different according to the Bonferroni Multiple Comparisons Test $(p<0.05)$.

The kinetics of degradation of AFB1 over the first $16 \mathrm{~h}$ incubation with Actinomycetes cultures at $30{ }^{\circ} \mathrm{C}$ and $\mathrm{pH} 6.0$ is shown in Figure 5. There was no significant difference in the ability of each of the microorganisms to degrade AFB1 with increasing time. The rate of degradation by $R$. erythropolis ATCC 4277 was higher compared to the other two strains. After two and a half hours, 50\% of AFB1 degraded, whereas $70 \%$ and $90 \%$ remained when AFB1 was exposed to S. lividans TK 24 and S. aureofaciens ATCC 10762 cultures, respectively. A significant difference $(p<0.05)$ was found between negative control and the treated samples. The control was stable over a period of $72 \mathrm{~h}$. Comparable results were determined by Teniola et al. (2005) [19]. Their results indicate that $90 \%$ was degraded after only $4 \mathrm{~h}$ incubation with cell-free extract of $R$. erythropolis, whilst after $8 \mathrm{~h}$ AFB1 was not detectable. Furthermore, culture supernatant of $S$. maltophilia showed high degradation activity where about $78.8 \%$ was degraded after $72 \mathrm{~h}$ incubation [21].

\subsection{Confirmation of AFB1 Degradation}

Four techniques were used to confirm the degradation: TLC, Reverse phase HPLC, ion-trap ESIMS, and high resolution FTMS (HR-FTMS). The TLC result confirmed AFB1 degradation. The TLC showed clearly the cleavage of the lactone group. The amount of AFB1 fluorescence decreased within the first $24 \mathrm{~h}$ and disappeared after $72 \mathrm{~h}$ of incubation with Actinomycetes. However, it is known that opening 
the difuran ring will not affect the molecules' fluorescence, while opening or abolishing the lactone ring does affect the fluorescence. Lee et al. (1981) [28] reported that AFB1 fluorescence is associated with the presence of an intact lactone ring as well as Motomura et al. (2003) [11] who reported that the decrease of AFB1 fluorescence indicated cleavage of the lactone ring. In this research, the Reversed phase HPLC also showed a decrease in the fluorescence intensity within the time, and a smaller peak was detected after $72 \mathrm{~h}$ of incubation with Actinomycetes. However, HPLC and TLC analysis did not reveal the formation of any breakdown products. Therefore, a more sensitive technique was required to confirm the degradation and to identify the breakdown products.

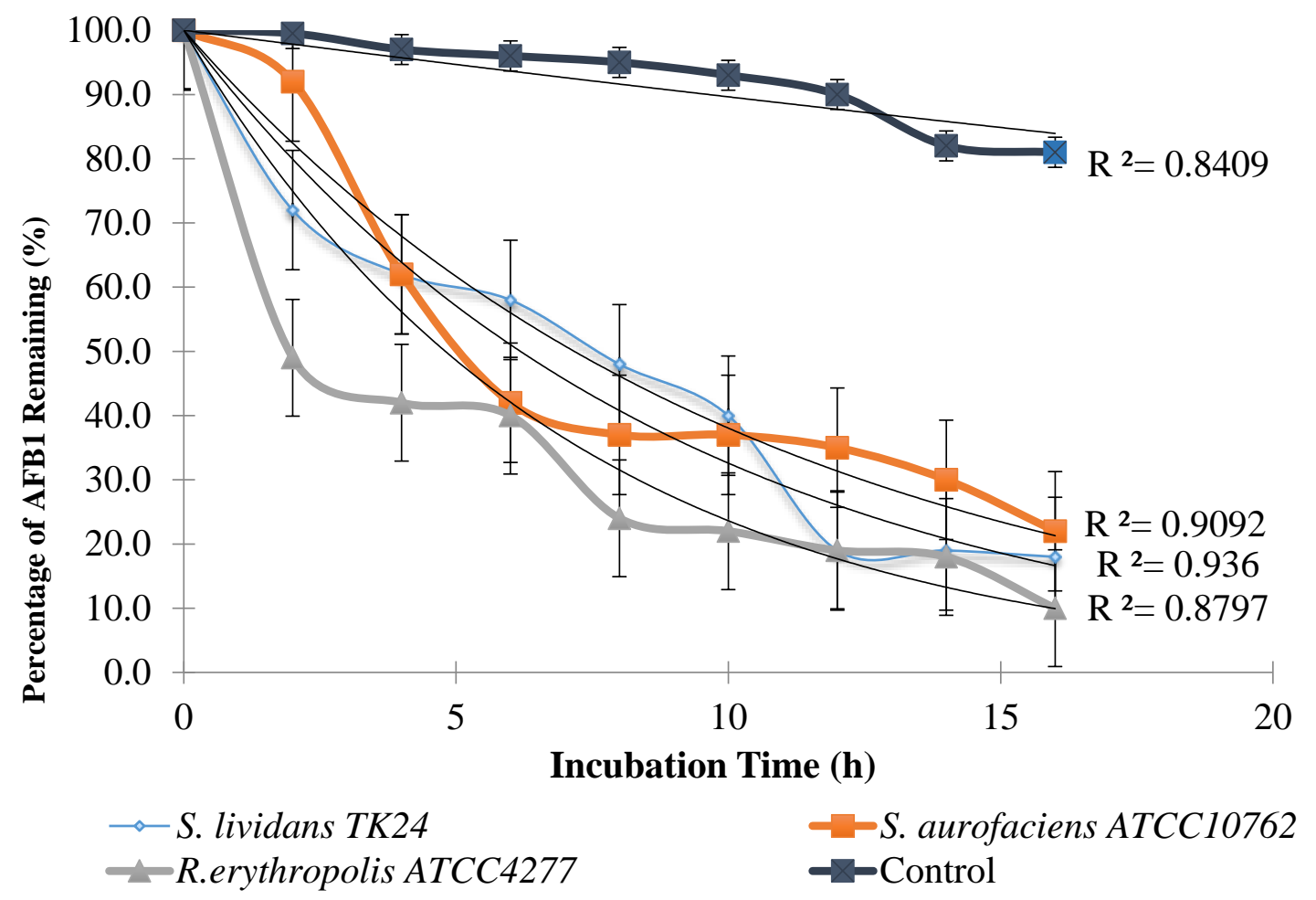

Figure 5. Kinetics of degradation of AFB1 over the first $16 \mathrm{~h}$ of incubation at $\mathrm{pH} 6$.

\subsection{Breakdown Product Identification and AFB1 Degradation Hypothesis}

The third aim of this study was to define a possible degradation mechanism of AFB1 by the microbial liquid cultures used in this research. The HPLC, ion-trap ESIMS, and HR-FTMS confirmed the cleavage of the lactone group, as the peak area designated for AFB1 was decreasing over time. Meanwhile, the LC-ESIMS indicated that another metabolite was being produced during AFB1 degradation. These results were consistent with the HR-FTMS results. The similarity and diversity of the samples at different incubation times were determined by principal component analysis (PCA) where similar samples were clustered (Figure 6). The result showed that the metabolites were clustered together according to each microorganism used. There was no significant difference in the ability of each of the microorganisms to degrade AFB1 with increasing time. However, it was also observed that the metabolites for $R$. erythropolis at 48 and $72 \mathrm{~h}$ clustered separately from the other two microorganisms. Greater emphasis was given to $R$. erythropolis as it was the most efficient in terms of AFB1. 


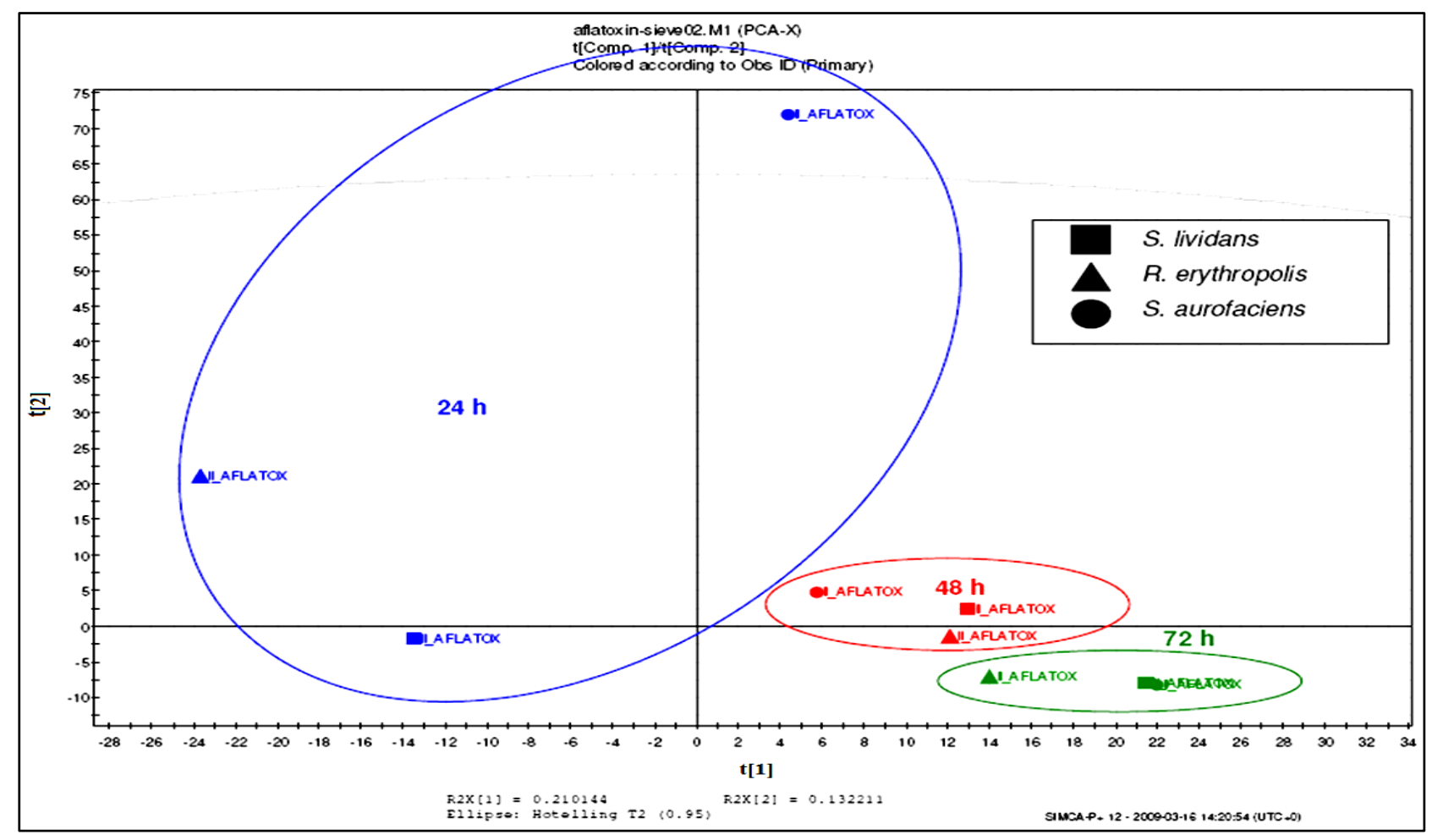

Figure 6. PCA and Multivariate data analysis of AFB1 by different Actinomycete strains at variable time frames.

The high resolution FT mass spectroscopy LTQ-Orbitrap was utilised to confirm AFB1 degradation as well as to identify any of the metabolites produced during the biodegradation process. The mass spectral data from the Orbitrap were processed and analysed using the SIEVE software. The latter identified new mass ion peaks of other metabolites at $\mathrm{nM}$ concentrations. Treated samples were compared with the control at zero time. Significant ion peaks found in the treated samples were detected by SIEVE analysis including $[\mathrm{M}+\mathrm{H}]^{+}$ion peaks at $\mathrm{m} / \mathrm{z} 331.2845,287.2219$ and 237.1121 (Figure 7) as well as the presence of other smaller molecules. These catabolic products were achieved during the $R$. erythropolis culture treatment. The formation of catabolic products, together with the presence or absence of AFB1, was evaluated by PCA analysis. It was observed that metabolites for $R$. erythropolis at 48 and $72 \mathrm{~h}$ clustered separately from the other two microorganisms. In addition, as generated by the SIEVE data which is shown in Figure 8, the $[\mathrm{M}+\mathrm{H}]^{+}$ion peak at $\mathrm{m} / z 237.1121$ was being produced over a period of $72 \mathrm{~h}$. It is clear that the concentration of this particular metabolite increased as AFB1 decreased.

SIEVE analysis and the correlation study identified which compounds were related to AFB1 degradation (Table 1). Positive and negative correlations were found between AFB1 and marker metabolites generated during the degradation process. 

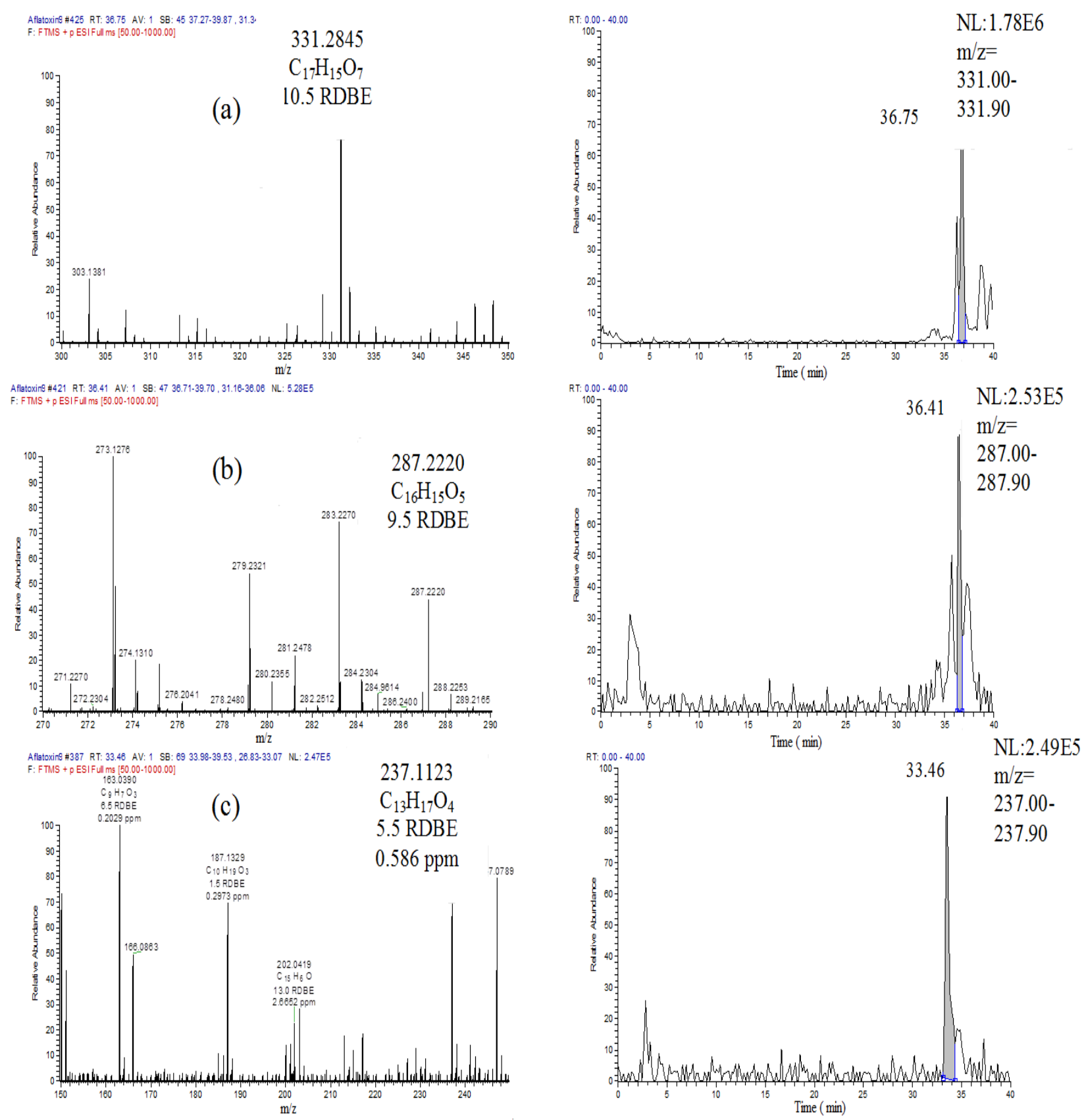

Figure 7. MS spectral data for $[\mathrm{M}+\mathrm{H}]^{+}$ion peaks at $\mathrm{m} / \mathrm{z} 331.2845(\mathbf{a}), 287.2220(\mathbf{b})$ and 237.1121 (c) with each of their extracted ion chromatogram after $72 \mathrm{~h}$ of incubation time with $R$. erythropolis culture.

A highly negative correlation was found for the ion peak at $\mathrm{m} / z 237.1121$ indicating a significant effect ( $p$ < 0.05) on AFB1 degradation (see Table 1), as the peak area of AFB1 was decreasing, and the total ion peak area for $\mathrm{m} / \mathrm{z} 237.112$ was increasing. AFB1 was degraded to a smaller metabolite of $236 \mathrm{amu}$ with the suggested molecular formula of $\mathrm{C}_{13} \mathrm{H}_{16} \mathrm{O}_{4}$. Interestingly, over a period of $72 \mathrm{~h}$ incubation time, an increase of 236 amu metabolite was also associated with an increase in the fatty acid and glycophosphate compounds, as shown by the SIEVE-generated data, assisted with identified hits from the KEGG (Kyoto Encyclopedia of Genes and Genomes) pathway database (Figure 9). This also suggests the degradation of AFB1 to low molecular weight compounds prior to their plausible participation in the citrate cycle. 


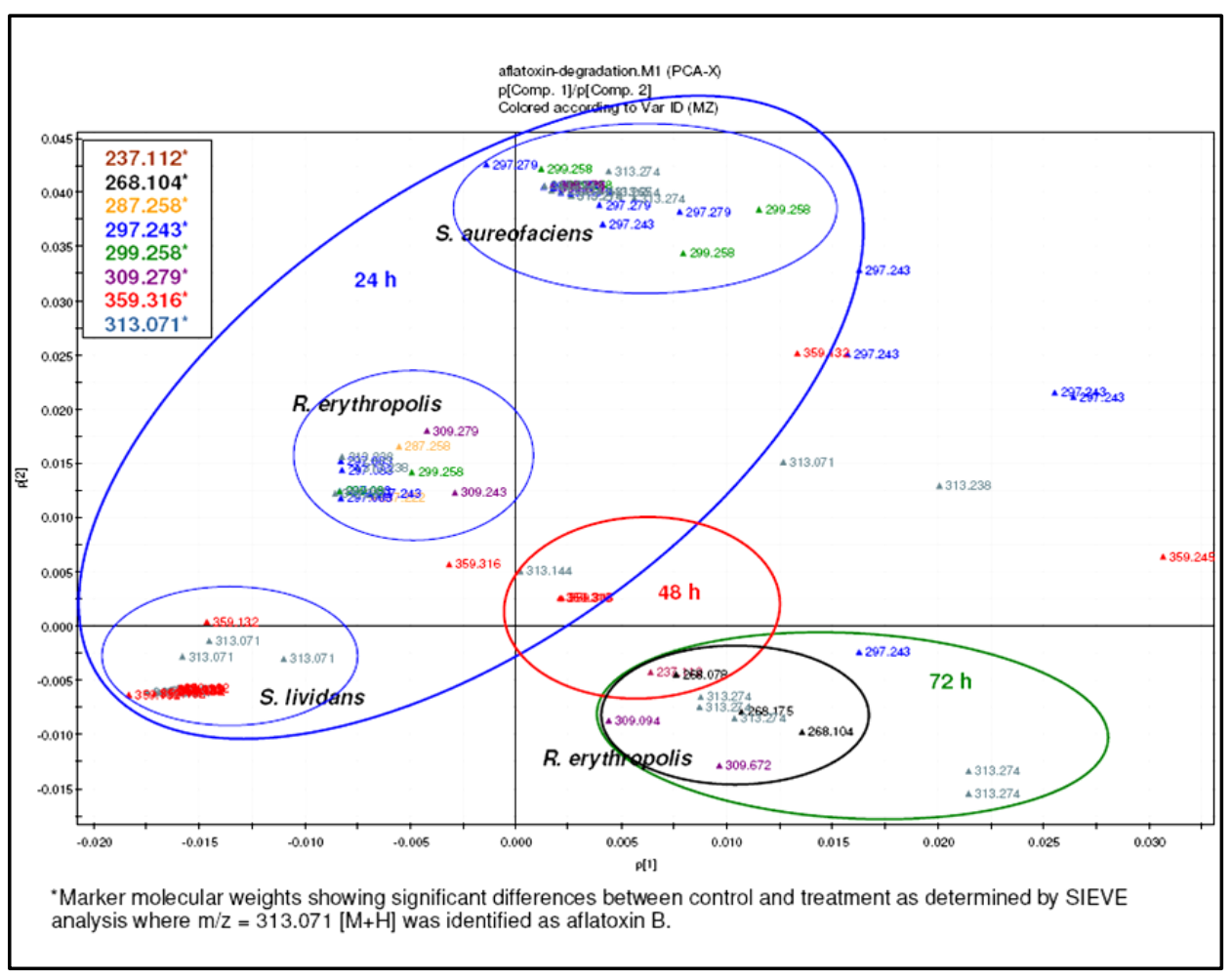

Figure 8. PCA analysis of AFB1 degradation by different Actinomycete strains showing the marker metabolites.

Table 1. Positive and negative correlation of marker metabolites generated during the degradation process of AFB1.

\begin{tabular}{cccc}
\hline $\boldsymbol{m} / \boldsymbol{z}$ & Peak area & Correlation & $\boldsymbol{p}$ value \\
\hline 331.2845 & 782442 & -0.921 & 0.254 \\
297.2428 & 2418033 & -0.546 & 0.633 \\
299.2584 & 117588 & 0.809 & 0.400 \\
287.2219 & 115295 & -0.645 & 0.263 \\
269.2477 & 1488 & 0.916 & 0.554 \\
237.1121 & 31685 & -0.998 & 0.041 \\
\hline
\end{tabular}

Our hypothesis involves the degradation of AFB1 into another compound with chemical properties different from AFB1. Therefore, the HPLC run was set for 40 min to account for the formation of new metabolites. Furthermore, AFB1 degradation involves conversion of AFB1 into other analogues. High resolution FTMS results in this research were indicative of the formation of new metabolites along with the degradation of AFB1. The mass spectrum of the treated sample showed intense pseudomolecular ion peak values at $m / z, 331.0707,287.2219,237.1211$ as well as that of at $\mathrm{m} / \mathrm{z} 313.0707$ attributable to residual AFB1. These ions were not present in the mass spectrum of either the reference sample of pure AFB1 or in the control samples. As a result, these metabolites were inferred as degradants achieved during the culture treatment. Consequently, the hypothesis for AFB1 degradation may involve the formation of the $\beta$-keto acid structure catalysed by enzymes produced by $R$. erythropolis, followed by hydrolysis of the lactone ring resulting in a metabolite with $330 \mathrm{amu}$ (3.16-II). The hydrolysis was followed by decarboxylation of the open lactone ring yielding to $286 \mathrm{amu}$ (3.16-III), which is known as 
AFD1. This involved the formation of $206 \mathrm{amu}$ (3.16-IV), which is AFD2, where the difuran moiety was retained while the lactone carbonyl and cyclopentenone ring characteristic of an AFB1 molecule disappears. The enzymatic procedure involved cleavage of the unsaturated part of one difuran unit (3.16-V) yielding a furanolactone phenolic metabolite of $236 \mathrm{amu}$ (3.16-VI) (Figure 10).

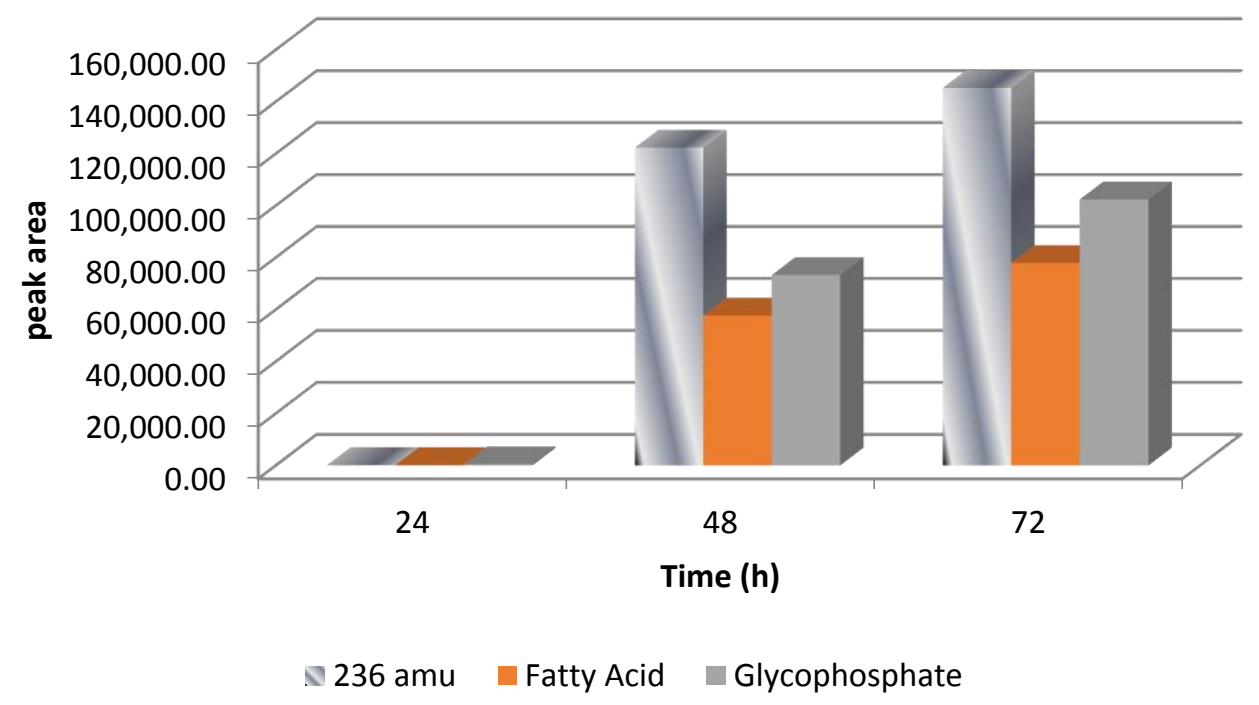

Figure 9. Summary of SIEVE-generated data for the formation of $[\mathrm{M}+\mathrm{H}]^{+}$ion peak at $\mathrm{m} / \mathrm{z}$ 237.1121 along with fatty acid and glycophosphate content over a period of $72 \mathrm{~h}$ with R. erythropolis culture.

An early study indicated that AFB1 was most likely metabolised to degradation products with chemical properties different from those of AFB1, but they could not reveal the formation of any breakdown products through electron spray mass spectrometry. In another work, chemical inactivation of $\mathrm{AFB} 1$ and aflatoxin $\mathrm{B}_{2}\left(\mathrm{AFB}_{2}\right)$ in maize grain by means of $1 \mathrm{~N}$ aqueous citric acid was reported [29,30]. Suttajit (2015) [31] suggested a complete degradation for AFB1 by chemical degradation; the hypothesis involved the use of high temperatures $\left(220-340{ }^{\circ} \mathrm{C}\right)$ and ammonia. There are few studies on AFB1 biodegradation, and none of them show a complete degradation mechanism. Most of the proposed mechanisms in the biological system involved biotransformation of AFB1 into its derivatives.

Motomura et al. (2003) suggested that the specific enzyme cleaved the lactone ring of AFB1, although the degradation products of AFB1 were not investigated clearly; as the technique used was limited, they could not reveal any intermediate products [11]. In other research, a multi-enzyme from Armillariella tabescense was isolated and purified. These enzymes were capable of degrading AFB1 where the optimum activity was at $35^{\circ} \mathrm{C}, \mathrm{pH}$ 6.8. Their proposed pathway indicated the degradation of AFB1 by multi-enzymes: AFB1 was first transformed to its epoxide, followed by hydrolysis of the epoxide to give a dihydrol. Then, the difuran ring would open in the subsequent hydrolysis step [6]. However, the conversion of AFB1 to its epoxide is unlikely as it is more toxic and carcinogenic. Alberts et al. (2006) [32] and Teniola et al. (2005) [19] suggested that Rhodococcus could use a cascade of enzyme reactions to degrade AFB1 without suggesting any hypothesis for the degradation. Furthermore, Lapalikar et al. (2012) [33] identified and characterised enzymes from $R$. erythropolis that could degrade AFB1 into smaller compounds. 
In this study, the interesting results were detected by HR-FTMS analysis, suggesting that AFB1 degradation is associated with the accumulation of intermediates of fatty acid metabolism, and glycolysis. Various enzymes were identified from R. erythropolis involving catabolic pathways of aromatic compounds such as polychlorinated biphenyls enzymes, which included ring cleavage, biphenyl dioxygenases, dihydrodiol dehydrogenase and hydrolases [10]. Furthermore, all the central pathways for aromatic degradation ended with the citrate cycle [10]. AFB1 is a polyaromatic compound, and the degradation may occur in the same pathway as mentioned for the aromatic compound. However, more investigation is required to identify the enzyme or enzyme systems responsible for the biodegradation.<smiles>COc1cc2c(c3oc(=O)c4c(c13)CCC4=O)C1C=COC1O2</smiles>

I

Chemical Formula: $\mathrm{C}_{17} \mathrm{H}_{12} \mathrm{O}_{6}$ Exact Mass: 312.0634<smiles>COc1cc(O)c2c(c1)OC1OC=CC21</smiles>

IV

Chemical Formula: $\mathrm{C}_{11} \mathrm{H}_{10} \mathrm{O}_{4}$ Exact Mass: 206.0579<smiles>C=C=CC1OC2Oc3cc(OC)c(C4=C(C(=O)O)C(=O)CC4)c(O)c3C12</smiles>

II

Chemical Formula: $\mathrm{C}_{17} \mathrm{H}_{14} \mathrm{O}_{7}$ Exact Mass: 330.0740<smiles>[C]=C</smiles><smiles>C=C=C1C=CC2c3c(cc(OC)c(C4=CC(=O)CC4)c3O)OC2O1</smiles>

III

Chemical Formula: $\mathrm{C}_{16} \mathrm{H}_{14} \mathrm{O}_{5}$ Exact Mass: 286.0841

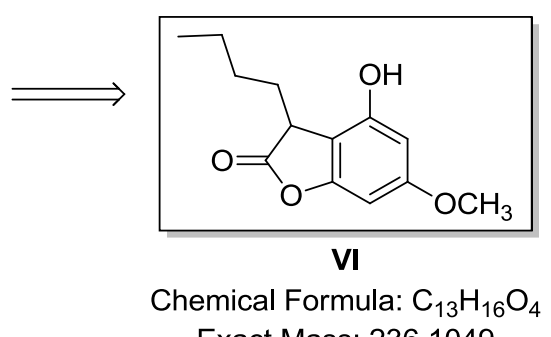

Figure 10. Hypothetical degradation mechanism for AFB1 by R. erythropolis.

\section{Experimental Section}

\subsection{Microbial Strains and Cultivation}

Biological degradation of AFB1 using three different strains of Actinomycetes was investigated in this study. The $R$. erythropolis strain was selected based on published data [19] while the Streptomyces strains were tested against AFB1 for the first time. Strains of (Streptomyces lividans TK 24 and 
Streptomyces aureofaciens ATCC10762) used in this study were obtained from the Institute of Pharmacy and Biomedical Sciences (SIPBS) collection at the University of Strathclyde, Glasgow, UK. R. erythropolis ATCC 4277 was purchased from (ATCC LGC Standards, Bury, UK). The strains were cultivated in Difco ISP medium No.1 comprised of $0.5 \%(\mathrm{w} / \mathrm{v})$ pancreatic digest of casein and $0.3 \%$ $(w / v)$ yeast extract (Sigma Aldrich, Dorset, UK). The cultures were stored at $-80{ }^{\circ} \mathrm{C}$ in $20 \%$ glycerol until use. Prior to use, the strains were inoculated into $250 \mathrm{~mL}$ of Difco ISP medium No. 1 and incubated at $30{ }^{\circ} \mathrm{C}$ for $48 \mathrm{~h}$ and pH 6.0 at $200 \mathrm{rpm}$ on a shaker (New Brunswick Scientific, Edison, NJ, USA).

\subsection{AFB1 Degradation by Actinomycetes in Liquid Culture}

$0.2 \mathrm{~mL}$ of a $100 \mu \mathrm{g} / \mathrm{mL} \mathrm{AFB}_{1}$ dissolved in methanol (Sigma Aldrich, Dorset, UK.) was added to $2 \mathrm{~mL}$ Eppendorf tubes. The methanol evaporated before adding the culture. Then $50 \mu \mathrm{L}$ of $48 \mathrm{~h}$ pre inoculums were inoculated into $0.75 \mathrm{~mL}$ of Difco ISP medium No. 1, adding to $2 \mathrm{~mL}$ sterile screw-capped Eppendorf tubes to a final concentration of $20 \mu \mathrm{g} / \mathrm{mL}$. The cultures were incubated at $30{ }^{\circ} \mathrm{C}$ for 24,48 , and 72 hours. Microbial growth was monitored by optical density observations of the cell suspension at $600 \mathrm{~nm}$. The cells were removed by centrifugation (JuanBR4i Multifunction, ThermoScientific, Porton Down, UK) at $11,000 \mathrm{rpm}$ for $15 \mathrm{~min}$ at $4^{\circ} \mathrm{C}$. AFB1 was quantified by using reversed phase HPLC as described below. Sterile medium supplement with AFB1 with a final concentration of $20 \mu \mathrm{g} / \mathrm{mL}$ was used as control.

\subsection{Optimization of the Degradation Conditions}

The degradation of AFB1 at different temperatures, $\mathrm{pH}$ and incubation times was investigated to determine the optimum condition for degradation by the different strains. The chosen temperatures were $25,30,35$, and $40^{\circ} \mathrm{C}$ over a period of $24 \mathrm{~h}$ at $\mathrm{pH}$. The $\mathrm{pH}$ values used in this experiment were $4,5,6$, 7 , and 8 . The cultures were incubated in the dark for $24 \mathrm{~h}$ at $30{ }^{\circ} \mathrm{C}$. The $\mathrm{pH}$ was adjusted by using $1 \mathrm{~N}$ $\mathrm{HCI}$ or $1 \mathrm{~N} \mathrm{NaOH}$ as required. The control was used in this study (media+AFB1 $20 \mu \mathrm{g} / \mathrm{mL}$ ) and AFB1 was quantified by using reversed phase HPLC as described below. All experiments were performed in triplicates.

\subsection{Quantification of AFB1 and Its Degradants}

Extracting AFB1 from the medium was not necessary as one of the study aims was to purify and identify the degradants. Four methods were used in this study to confirm AFB1 degradation: Thin layer chromatography (TLC) (Sigma Aldrich, Dorset, Gillingham, UK), High-performance liquid chromatography (HPLC) (Gilson instrument, Luton, Bedfordshire, UK), Liquid chromatography-mass spectrometry (LCMS) (ThermoFinnigan, Bremen, Germany) and high resolution Fourier transform and orbitrap mass spectrometry (Orbitrap FTMS) (ThermoFinnigan, Bremen, Germany).

\subsubsection{TLC}

TLC analysis was done on silica gel ( $\left.\mathrm{Si} \mathrm{6}_{0}\right)$ plates (Sigma Aldrich, Dorset, Gillingham, UK). The plates were developed using chloroform: acetone $(9: 1, v / v)$ as solvent system and monitored under UV at $365 \mathrm{~nm}$. 


\subsubsection{Reversed phase HPLC}

Reversed phase HPLC (Gilson instrument) analysis was performed through a RP-C18 (5 $\mu$ m, $150 \times 4.6 \mathrm{~mm}$ ID) column (ACE, Hichrom limited, West Berkshire, UK) attached to a C6-phenyl $4.0 \times 3.0 \mathrm{~mm}$ ID guard column (Phenomenex, Macclesfield, Cheshire, UK). Elution was achieved at a flow rate of $1 \mathrm{~mL} / \mathrm{min}$ with acetonitrile: methanol: water $(1: 1: 2, v / v / v)$ as the mobile phase. Injection volume was $20 \mu \mathrm{L}$. The sample temperature was controlled at $40{ }^{\circ} \mathrm{C}$ by using column heater model 7971 (Jones chromatography, Mid Glamorgan, UK). A photo-diode array detector (PDA) was used to monitor the presence of AFB1 by UV detection (Gilson, Luton, Bedfordshire, UK) at a wavelength of $365 \mathrm{~nm}$. The data were collected and processed with Gilson unipoint LC system software (Gilson, Luton, Bedfordshire, UK). A control was used in this study (media + AFB1 $20 \mu \mathrm{g} / \mathrm{mL}$ ). The percentage of remaining AFB1 was calculated using the following formula:

$$
\text { Percentage of AFB1 Remaining }=\left(\frac{\text { AFB1 peak area in the treatment }}{\text { AFB1 peak area in control }}\right) \times 100
$$

\subsubsection{LCMS}

The LCMS procedure was used to further confirm that AFB1 degradation was performed on a HPLC-PDA Agilent 1100 system coupled with the LCQ Deca XP (ThermoFinnigan, Bremen, Germany) ion trap instrument equipped with an electrospray ionization (ESI) source. Separation was accomplished on a reversed phase ACE 5 C18-300 $5 \mu \mathrm{m}, 300 \AA$, $30 \times 4.6$ ID mm column (Hichrom Limited, Reading, UK). The mobile phase consisted of acetonitrile: methanol: water $(1: 1: 2, v / v / v)$ with $0.01 \mathrm{M}$ of ammonium formate buffer at a flow rate of $0.4 \mathrm{~mL} / \mathrm{min}$. UV and mass spectral data were recorded for $5 \mathrm{~min}$. ESI mass spectra ranging from $\mathrm{m} / \mathrm{z} 50$ to $1000 \mathrm{amu}$ were taken in the positive-ion mode. The pseudo-molecular ion peak at $m / z 313[\mathrm{M}+\mathrm{H}]^{+}$for AFB1, was monitored through its $\mathrm{MS}^{2}$ data. The MS detector (ThermoFinnigan, Bremen, Germany) was set at a vaporizer temperature of $220{ }^{\circ} \mathrm{C}$; a sheath gas flow rate of 50 arbitrary units; auxiliary gas flow rate of 10 arbitrary units; source voltage was at $5 \mathrm{kV}$; capillary voltage at $15 \mathrm{~V}$; and the tube lens offset at $30 \mathrm{~V}$. Two scan events were run; the first was the full scan range from $\mathrm{m} / \mathrm{z}, 50$ to 1000 in positive mode and the second run was an $\mathrm{MS}^{2}$ fragmentation for $m / z 313$ set at the range of 85-500 amu at the same mode. A control was used in this study (media + AFB1 $20 \mu \mathrm{g} / \mathrm{mL}$ ), and the mass spectral data were processed with Xcalibur 2.0 (ThermoFinnigan, Bremen, Germany).

\subsubsection{LTQ-Orbitrap}

The high resolution Fourier transform mass spectroscopy technique was achieved with the LTQ-Orbitrap to identify the metabolomes produced during the biodegradation process. The LTQ-Orbitrap (ThermoFinnigan, Bremen, Germany) instrument equipped with an ESI source was coupled to a Surveyor HPLC-PDA (ThermoFinnigan, Bremen, Germany) system. The HPLC conditions were identical to those employed on the LCQ Deca XP instrument. However, a gradient run was used, commencing with 5\% acetonitrile for $5 \mathrm{~min}$ and increased to $100 \%$ after $35 \mathrm{~min}$ with an additional $5 \mathrm{~min}$ at $100 \%$ acetonitrile. Spectral data were recorded for the entire $40 \mathrm{~min}$. AFB1 was detected and eluted at $12.5 \mathrm{~min}$. The MS detector was set at a vaporizer temperature of $220^{\circ} \mathrm{C}$; a sheath gas flow rate of 
30 arbitrary units; auxiliary gas flow rate of 10 arbitrary units; the source voltage was at $4 \mathrm{kV}$; the capillary voltage was at $35.5 \mathrm{~V}$, the tube lens at $30 \mathrm{~V}$; and the capillary voltage was at $21 \mathrm{~V}$. The data were processed by Xcalibur 2.0 while metabolomic studies were done using SIEVE 1.2 (ThermoFinnigan, Bremen, Germany), differential analysis software to assist peak monitoring at lower thresholds. The SIEVE software was connected with the online library ChemSpider to detect aflatoxin analogues and other smaller molecule degradants. The same set of data generated by SIEVE was exported to SIMCA P + 12 (Umetrics, Umeå, Sweden) for principal component analysis to determine any similarity and differences in metabolome formation during the degradation process by the different strains at different incubation times. Two controls were used (media + AFB1 20 ia + ols were used d at 12.5 min. The AFB1 was used as a blank control to enable the exclusion of background mass ion peaks belonging to the culture media.

\section{Statistical Analysis}

Data on the effect of the $\mathrm{pH}$, temperature and time on the response variable AFB1 concentration were statistically evaluated using one and two-way ANOVA. The results were considered significant when $p$ values were $p<0.05$. Tukey's multiple comparison tests were done when significant difference was encountered. In addition, multivariate analysis using SIMCA was used to analyze the data. The purpose of using SIMCA is to determine the similarity of a group of samples according to their principle component where each group is described as a cluster. Furthermore, a correlation between AFB1 degradation and certain metabolites was analysed with Minitab software.

\section{Conclusions}

In this study, biological degradation of AFB1 by 3 Actinomycete species, (Rhodococcus erythropolis ATCC 4277, Streptomyces lividans TK 24, and S. aureofaciens ATCC 10762) was examined in liquid cultures. The degradation of AFB1 by these cultures was achieved without pre-exposing the cultures to the toxin. Our study extended the range of microorganisms capable of degrading AFB1. No significant difference was found between the three cultures in terms of ability to degrade AFB1 over a period of $72 \mathrm{~h}$. The degradation was temperature, time and $\mathrm{pH}$ dependent. However, each microorganism has a different way of degrading AFB1. The metabolites produced during AFB1 degradation by $R$. erythropolis were significantly different from those produced during degradation by the other two microorganisms. The research gained new insight into the biodegradation pathway by Rhodococcus and elucidated the factors that influence metabolite degradation. TLC assay confirmed the cleavage of the lactone group by Rhodococcus. A hypothetical degradation mechanism for AFB1 by R. erythropolis was proposed in this study based on our result and linked to the published work of others. The degradation of AFB1 was associated with the increase of fatty acid and glycophosphate metabolites. Our findings provide valuable information that can be used to devolve a model for industrial fermentation for food processing or bioenergy production. More work is still required to gain a better understanding of AFB1 degradation by these microorganisms. Further investigation of toxicity and enzyme identification and purification involved in toxin biodegradation by Actinomycetes is undergoing research. 


\section{Acknowledgments}

The Authors would like to thank Farage Al-Ghazzewi for reviewing this manuscript pre-submission. The financial support of this research by the Ministry of Libyan Higher Education; Tripoli; Libya is gratefully acknowledged.

\section{Author Contributions}

Manal Eshelli conceived, designed, performed the experiments, analyzed the data and wrote the manuscript. RuAngelie Edrada-Ebel contributed in LCMS, LTQ-Orbitrap and SIMCA analysis. Linda Harvey and Brian McNeil supervised the experiments.

\section{Conflicts of Interest}

The authors declare no conflict of interest.

\section{References}

1. Bennett, J.W.; Klich, M. Mycotoxins. Clin. Microbiol. Rev. 2003, 16, 497-516.

2. Medeiros, F.H.V.D.; Martins, S.J.; Zucchi, T.D.; Melo, I.S.D.; Batista, L.R.; Machado, J.D.C. Biological control of mycotoxin-producing molds. Ciênc. Agrotecnol. 2012, 36, 483-497.

3. Bolognani, F.; Rumney, C.J.; Rowland, I.R. Influence of carcinogen binding by lactic acid-producing bacteria on tissue distribution and in vivo mutagenicity of dietary carcinogens. Food Chem. Toxicol. 1997, 35, 535-545.

4. Kabak, B. The fate of mycotoxins during thermal food processing. J. Sci. Food Agric. 2009, 89, 549-554.

5. El-Nezami, H.; Kankaanpaa, P.; Salminen, S.; Ahokas, J. Ability of dairy strains of lactic acid bacteria to bind a common food carcinogen, aflatoxin B-1. Food Chem. Toxicol. 1998, 36, 321-326.

6. Liu, D.L.; Yao, D.S.; Liang, R.; Ma, L.; Cheng, W.Q.; Gu, L.Q. Detoxification of aflatoxin B-1 by enzymes isolated from Armillariella tabescens. Food Chem. Toxicol. 1998, 36, 563-574.

7. Kusumaningtyas, E.; Widiastuti, R.; Maryam, R. Reduction of aflatoxin B1 in chicken feed by using Saccharomyces cerevisiae, Rhizopus oligosporus and their combination. Mycopathologia 2006, 162, 307-311.

8. Wu, Q.; Jezkova, A.; Yuan, Z.; Pavlikova, L.; Dohnal, V.; Kuca, K. Biological degradation of aflatoxins. Drug Metab. Rev. 2009, 41, 1-7.

9. Liu, D.L.; Ma, L.; Gu, L.Q.; Liang, R.; Yao, D.S.; Chen, W.Q. Armillariella Tabescen Enzymatic detoxification of aflatoxin B1: Part III. Immobilized enzymatic detoxification. In Proceedings of the 14th International Enzyme Engineering Conference, Beijing, China, 12-17 October 1997; Laskin, A.I., Li, G.X., Yu, Y.T., Eds.; Annals of the New York Academy of Sciences: New York, NY, USA, 1997; pp. 592-599.

10. Martinkova, L.; Uhanakova, B.; Patek, M.; Nesvera, J.; Kren, V. Biodegradation potential of genus Rhodococcus. Environ. Int. 2009, 35, 162-177.

11. Motomura, M.; Toyomasu, T.; Mizuno, K. Shinozawa Purification and chracterization of an aflatoxin degrdation enzyme from Pleurotus ostreatus. Microbiol. Res. 2003, 158, 237-242. 
12. Varga, J.; Toth, B. Novel strategies to control mycotoxins in feeds: A review. Acta Vet. Hung. 2005, 53, 189-203.

13. Shantha, T. Fungal degradation of aflatoxin B1. Nat. Toxins 1999, 7, 175-178.

14. Shetty, P.H.; Jespersen, L. Saccharomyces cerevisiae and lactic acid bacteria as potential mycotoxin decontaminating agents. Trends Food Sci. Technol. 2006, 17, 48-55.

15. Bueno, D.J.; Casale, C.H.; Pizzolitto, R.P.; Salano, M.A.; Olivier, G. Physical Adsorption of Aflatoxin B1 by lactic acid bacteria and Saccharomyces cerevisiae: A theoretical model. J. Food Prot. 2006, 7, 2148-2154.

16. El-Nezami, H.; Mykkanen, H.; Kankaanpaa, P.; Salminen, S.; Ahokas, J. Ability of Lactobacillus and Propionibacterium strains to remove aflatoxin B-1 from the chicken duodenum. J. Food Prot. 2000, 63, 549-552.

17. Elgerbi, A.M.; Aidoo, K.E.; Candlish, A.A.G.; Williams, A.G. Effects of lactic acid bacteria and bifidobacteria on levels of aflatoxin M-1 in milk and phosphate buffer. Milchwiss.-Milk Sci. Int. 2006, 61, 197-199.

18. El-Nezami, H.; Kankaanpaa, P.; Salminen, S.; Ahokas, J. Physicochemical alterations enhance the ability of dairy strains of lactic acid bacteria to remove aflatoxin from contaminated media. J. Food Prot. 1998, 61, 466-468.

19. Teniola, O.D.; Addo, P.A.; Brost, I.M.; Farber, P.; Jany, K.D.; Alberts, J.F.; van Zyl, W.H.; Steyn, P.S.; Holzapfel, W.H. Degradation of aflatoxin B-1 by cell-free extracts of Rhodococcus erythropolis and Mycobacterium fluoranthenivorans sp. nov. DSM44556(T). Int. J. Food Microbiol. 2005, 105, 111-117.

20. Cserháti, M.; Kriszt, B.; Krifaton, C.; Szoboszlay, S.; Háhn, J.; Tóth, S.; Nagy, I.; Kukolya, J. Mycotoxin-degradation profile of Rhodococcus strains. Int. J. Food Microbiol. 2013, 166, $176-185$.

21. Guan, S.; Ji, C.; Zhou, T.; Li, J.X.; Ma, Q.G.; Niu, T.G. Aflatoxin B-1 degradation by Stenotrophomonas maltophilia and other microbes selected using coumarin medium. Int. J. Mol. Sci. 2008, 9, 1489-1503.

22. Kong, Q.; Zhai, C.; Guan, B.; Li, C.; Shan, S.; Yu, J. Mathematic modeling for optimum conditions on aflatoxin $\mathrm{B}_{1}$ degradation by the aerobic bacterium Rhodococcus erythropolis. Toxins $\mathbf{2 0 1 2}, 4$, 1181-1195.

23. Wang, F.Q.; Zhang, C.G.; Li, B.; Wei, D.Z.; Tong, W.Y. New Microbiological Transformations of Steroids by Streptomyces virginiae IBL-14. Environ. Sci. Technol. 2009, 43, 5967-5974.

24. Pogell, B.M. Bioremediation of pesticides and herbicides by Streptomycetes. In Proceedings of the International Symposium on Environmental Biotechnology, Waterloo, ON, Canada, 4-8 July 1994; MooYoung, M., Anderson, W.A., Chakrabarty, A.M., Eds.; Kluwer Academic Publ: Waterloo, ON, Canada, 1994; pp. 38-46.

25. Ciegler, A.; Lillehoj, E.B.; Peterson, R.E.; Hall, H.H. Microbial detoxification of Aflatoxin. Appl. Microbiol. 1966, 14, 934-939.

26. Richard, J.L. Some major mycotoxins and their mycotoxicoses-An overview. Int. J. Food Microbiol. 2007, 119, 3-10.

27. Smiley, R.D.; Draughon, F.A. Preliminary evidence that degradation of aflatoxin B1 by Flavobacterium aurantiacum is enzymatic. J. Food Prot. 2000, 63, 415-418. 
28. Lee, L.S.; Dunn, J.J.; DeLucca, A.J.; Ciegler, A. Role of lactone ring of aflatoxin B1 in toxicity and mutagenicity. Experientia 1981, 37, 16-17.

29. Mendez-Albores, A.; Arambula-Villa, G.; Loarea-Pina, M.G.F.; Castano-Tostado, E.; Moreno-Martinez, E. Safety and efficacy evaluation of aqueous citric acid to degrade B-aflatoxins in maize. Food Chem. Toxicol. 2005, 43, 233-238.

30. Mendez-Albores, A.; Veles-Medina, J.; Urbina-Alvarez, E.; Martinez-Bustos, F.; Moreno-Martinez, E. Effect of citric acid on aflatoxin degradation and on functional and textural properties of extruded sorghum. Anim. Feed Sci. Technol. 2009, 150, 316-329.

31. Suttajit, M. Mycotoxin prevention and control in foodgrains - Prevention and control of mycotoxins. Available online: http://www.fao.org/docrep/X5036E/x5036E0q.htm (accessed on 2 January 2015).

32. Alberts, J.F.; Engelbrecht, Y.; Steyn, P.S.; Holzapfel, W.H.; van Zyl, W.H. Biological degradation of aflatoxin B1 by Rhodococcus erythropolis cultures. Int. J. Food Microbiol. 2006, 109, 121-126.

33. Lapalikar, G.V.; Taylor, M.C.; Warden, A.C.; Scott, C.; Russell, R.J.; Oakeshott, J.G. $\mathrm{F}_{420} \mathrm{H}_{2}$-dependent degradation of aflatoxin and other furanocoumarins is widespread throughout the Actinomycetales. PLoS One 2012, 7, e30114.

(C) 2015 by the authors; licensee MDPI, Basel, Switzerland. This article is an open access article distributed under the terms and conditions of the Creative Commons Attribution license (http://creativecommons.org/licenses/by/4.0/). 